\title{
Hardware Architecture Review of Swarm Robotics System: Self-Reconfigurability, Self-Reassembly, and Self-Replication
}

\author{
Madhav Patil, Tamer Abukhalil, and Tarek Sobh \\ Computer Science and Engineering, University of Bridgeport, Bridgeport, CT 06604, USA \\ Correspondence should be addressed to Tamer Abukhalil; tabukhal@bridgeport.edu
}

Received 26 March 2013; Accepted 24 April 2013

Academic Editors: L. Asplund, D. K. Pratihar, and F. Torres

Copyright (C) 2013 Madhav Patil et al. This is an open access article distributed under the Creative Commons Attribution License, which permits unrestricted use, distribution, and reproduction in any medium, provided the original work is properly cited.

\begin{abstract}
Swarm robotics is one of the most fascinating and new research areas of recent decades, and one of the grand challenges of robotics is the design of swarm robots that are self-sufficient. This can be crucial for robots exposed to environments that are unstructured or not easily accessible for a human operator, such as the inside of a blood vessel, a collapsed building, the deep sea, or the surface of another planet. In this paper, we present a comprehensive study on hardware architecture and several other important aspects of modular swarm robots, such as self-reconfigurability, self-replication, and self-assembly. The key factors in designing and building a group of swarm robots are cost and miniaturization with robustness, flexibility, and scalability. In robotics intelligence, self-assembly and self-reconfigurability are among the most important characteristics as they can add additional capabilities and functionality to swarm robots. Simulation and model design for swarm robotics is highly complex and expensive, especially when attempting to model the behavior of large swarm robot groups.
\end{abstract}

\section{Introduction}

Over the past decade, an increasing number of research and development activities related to modular swarm robotics are attracting considerable attention and interest in industry and academia. This interest is inspired by, among other things, the emergent behavior observed in social insects such as ants, bees, wasps, and termites [1]. Self-reconfiguration, selfassembly, and self-replication are the main distinguishing characteristics of swarm robots, and a dream long held by many researchers in the field of robotics is to develop fully autonomous robotic systems with these characteristics [2]. As with many new technologies, this field is growing rapidly and becoming more complex, but there remains much to accomplish in the development of swarm robotics intelligence and swarm robotics hardware since the performance of a swarm robotics system depends greatly on its mechanical and electronic control design [3]. As a swarm multirobot system becomes more complex, each robot must still follow simple rules to perform a task or any application.

Swarm robot groups are usually homogeneous and controlled by a centralized or hierarchical system, depending on the application. Most of the robot platforms used in such swarm groups have the capability to assemble themselves according to the requirements of the task. Self-assembly is a process in which a group of swarm robots comes together to form a temporary large body structure capable of performing a job that is beyond the capability of single swarm robot [4]. Christensen, O'Grady, and Dorigo describe a robotic system that exhibits this kind of self-assembly. In this system, the basic units are themselves robots that can function either independently when disconnected from one another, or can function collectively when connected together to form a metastructure. The Christensen/O'Grady/Dorigo system demonstrates this kind of transformation of a collection of independent robots through a variety of different metastructure morphologies in physical hardware. Given enough units, if any individual unit in such a metastructure fails, the system would self-repair by replacing nonfunctional units with functional ones.

Self-reconfiguration is a process by which a robot metastructure constructed from physical structures or subsystems of [5] modular robots autonomously self-organizes and changes shape in order to adapt to different tasks or 


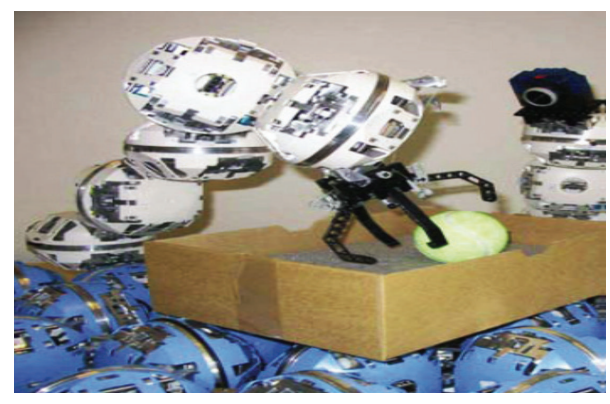

FIGURE 1: Lattice type architecture [20] (copyright @ 2007 Brandt et al.).

classes of terrain. For instance, some modular robots may transform into snakes in order to follow a tunnel and then may transform into quadrupeds to go upstairs. In self-reconfiguring, swarm modules are able to connect and disconnect without any human interaction as they offer such advantages as versatility, adaptability, robustness, and inexpensive production over traditional robots $[6,7]$. Due to these advantages, swarm robots exhibiting self-reconfigurability and self-assembly can be used to handle a wide range of tasks in an unknown or dynamic environment such as search and rescue operations after a fire or earthquake, undersea mining, planetary exploration, battlefield reconnaissance, and other application like service robotics and entertainment.

Fifty years ago, in 1947, Von Neumann proposed an automoton model sufficiently complex to reproduce itself [8-10]. Self-replication is another one of the characteristics of a modern swarm robot, in which several robot modules connect with each other to form an exact copy of the original robot [4]. The concept of kinematic self-reproduction has been applied in many research areas such as cellular automata, nanotechnology, macromolecular chemistry, and computer simulations. In the 1950 s and 1960s, Penrose presented the first implementation of a passive self-replicating machine. He showed that simple units or "bricks" having certain properties could be employed to build a self-reproducing machine under external agitation. The replicated robot function is the same as the original robot so that it can perform the same task. Only a few, high-level modules have successfully demonstrated the ability to self-replicate, primarily due to the great complexity of the process. Such a process is extremely challenging for low level modular robots.

This paper is organized as follows. First, we introduce the reader to background material and provide an overview of related work on swarm robotics hardware architecture. Next, we provide more details on sensors, actuation and manipulation, controllers and communication, and power options. Finally, we offer conclusions on our survey results.

\section{Swarm Robot: Self-Reconfigurability and Self-Replication}

In this section, we provide a brief survey of related work on swarm robot self-reconfiguration, self-replication, and self-reassembly. Modular robots are still in the process of becoming more flexible, autonomous, and more robust [11, 12]. Like any other robot, a swarm robot has two main organs, hardware and software. Software is the brain of the system, which gives a simulation environment to the functioning of the robot. The hardware brings directions stimulated by the software into action. When many such intercommunicating robots are deployed to work together, swarming action comes into play. However, only limited hardware platforms have been developed and used so far.

According to [12], self-reconfigurable robots are classified into three main types: chain, lattice, and mobile reconfiguration systems. In the chain and lattice types, each module typically remains connected to the (larger) modular robot at one or more points, while in mobile modular systems, the system self-reconfigures by having modules detach themselves from the modular robot and move independently to another location to reconnect. Self-reconfigurable robots have proven to be capable of self-repair $[13,14]$, self-assembly, and locomotion over a plane surface or over widely varied terrain [15].

Self-reconfiguration in a homogeneous system is simpler than in a heterogeneous system, but a heterogeneous swarm robot system might be more time efficient for accomplishing certain tasks. because the modular robot metastructure created by such a swarm system will be more compact due to the specialized capabilities of the modules [16].

Many sophisticated swarm intelligence robot platforms have been built to date by considering cost and functionality along with a flexible distributed intelligence structure. Some examples are as follows.

2.1. Lattice-Based Robot Architecture. In lattice architectures, the mobile robot units are connected and arranged in regular three dimensional cubic or hexagonal grid patterns. The lattice architecture offers relatively simpler reconfiguration and control, since motion is accomplished in parallel within an open loop framework. Homogeneous "molecubes" based on a lattice self-reconfigurable robot are demonstrated in [17]. Each "molecube" module is a $10 \mathrm{~cm}$ cube, and one half of it can swivel relative to the other half. Each half can bind with one additional module by using electromagnets. Latticebased self-reconfigurability and self-replication of a fourmodule entity are also demonstrated in [18] when the system is provided an ordered supply of additional units. The system executes a predetermined sequence of actions. ATRON is yet another lattice-based system, in which modules are arranged in a subset of a surface centered cubic lattice [19]. ATRON modules composed of two hemispheres are joined by a single revolute joint, as shown in Figure 1 [20]. In [20], Brandt, Christensen, and Lund discuss the mechanical design of ATRON and its resultant system properties, based on FEM analyses and real-world experiments. Fracta [21] and Metamorphic are also homogeneous 2-D lattice-based mechanical hardware characterized by hexagonally shaped robot modules. Other lattice-based robots like 3-D SRS, ICube [22], and Proteo [23] are also homogeneous in nature, which provides easy self-reconfiguration of these modules, but the hardware implementation is very complicated due to 


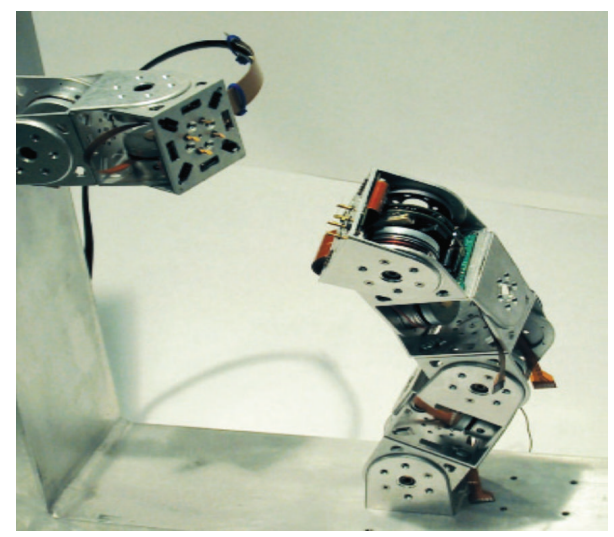

FIGURE 2: Chain type architecture [3] (copyright @ 2007 Yim et al.).

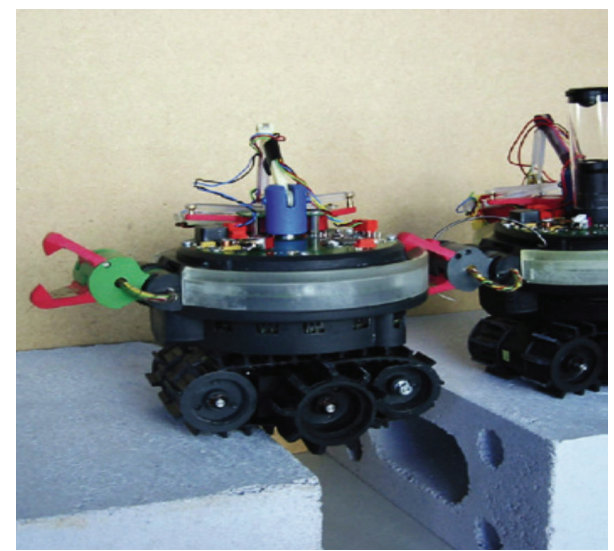

FIGURE 3: Mobile type architecture [35] (copyright @ 2003 Mondada et al.).

the geometric symmetry required for actuation and connection with other modules to provide more DOFs (므egrees $\underline{\text { Of }}$ Freedom).

2.2. Chain-Based Robot Architecture. Chain-based architectures have units that are connected together in a string or tree topology. The chain or tree can fold up physically to fill arbitrarily shaped spaces, but the underlying architecture is still serial. Through articulation, chain architectures potentially can reach any point or orientation in space and are therefore more versatile than some other architectures, but computationally they are more difficult to represent and analyze and hence are more difficult to control [3]. PolyBot $[13,24]$ is a modular chain robot that can configure its shape without human assistance. Yim et al. [3] have demonstrated the ability of PolyBots to self-reconfigure and self-reassemble with other PolyBots despite the limitation of each PolyBot to a single DOF, as shown in Figure 2. CONRO [25-27] is a homogeneous modular chain robot with a processor, power supply, sensors, and actuators on each module. The CONRO robot has demonstrated the capability of selfassembly. M-TRAIN [28] is another modular, distributed, self-reconfigurable homogeneous robot module which can change configuration by changing positions and connections with other M-TRAIN modules.

2.3. Mobile-Based Architecture. Mobile architectures have units that use the environment to maneuver around and can either hook up to form complex chains or lattices or form a number of smaller robots that execute coordinated movements and together form a larger "virtual" network. CEBOT [29] was proposed by Fukuda and Nakagawa with dynamically reconfigurable robotic systems and has heterogeneous modules with various different functions. CEBOT has gone through considerable development, and the later versions are called CEBOT Mark 1, 2, 3, and 4 [30]. CYBOT [31] is another type of a medium-powered mobile robot that is cheap enough to mass produce and hence assemble an interacting swarm. Gupta and Singh [32] proposed a low cost mobile module, the AUTOBOT robot, which can estimate the distance of obstacles and recognize multiple robots in an environment. The AUTOBOT module is capable of performing short-range communication using a $2.4 \mathrm{GHz}$ radio module and has two hours of battery backup power.

The S-BOT [33] is a fully autonomous, small wheeled cylindrical robot, $12 \mathrm{~cm}$ in diameter, $19 \mathrm{~cm}$ high, weighing approximately $700 \mathrm{~g}$, and is equipped with a variety of sensors. S-BOT's mobility is ensured by a differential drive system and mobile robot attachment architecture capable of clinging to other S-bots similar to itself by using a gripper. Dorigo [34] ran a set of experiments in which 18 S-bots demonstrated coordinated motion on rough terrain, hole and obstacle avoidance, self-assembly, cooperative transport, environmental exploration, and path formation. Recently, Swarm Bots (S-Bots) [35] have become one of the most popular swarm robot platforms because of their extreme plasticity, high degree of physical adaptation, and minimal requirement for human interaction and monitoring (Figure 3). IROBOT is another popular platform frequently used for swarm research. James McLurkin [36] describe work in which researchers implement an algorithm on a group of 25 IRobot SwarmBots and collect performance data. Each SwarmBot is mobile and has four IR transceivers at its corners, allowing communication with nearby robots and facilitating determination of the bearing, orientation, and ranges of its neighbors. A 32-bit microprocessor is used as a controller and all robots are homogeneous. Red, Blue, and Green LEDs and a MIDI audio system are used to provide audible and visual indications for monitoring the internal state of the robots.

Alice $[37,38]$ is a small rectangular mobile robot with dimensions of $22 \times 21 \mathrm{~mm}$, driven by two high efficiency SWATCH motors for locomotion, controlled by a PIC16F877 microcontroller with $8 \mathrm{~K}$ word of Flash EPROM program memory. Alice has four IR proximity sensors for obstacle detection, a short-range robot-to-robot communication system, and an IR receiver for remote control. Also, there are a wide variety of auxiliary modules for extending its capabilities, such as a linear camera, RF, and gripper modules.

E-puck [39] is a circular robot with a diameter of $70 \mathrm{~mm}$, driven by two stepper motors for locomotion, controlled by 
TABLE 1: Classification of swarm robots.

\begin{tabular}{|c|c|c|c|}
\hline System & Class & DOF & Reference(s) \\
\hline CEBOT & Mobile & Various & Fukuda et al. $[29,30]$ \\
\hline Polypod & Chain & 2 & $\operatorname{Yim}[3,12,13,23]$ \\
\hline Molecule & Lattice & 3 & Chirikjian et al. [3] \\
\hline CONRO & Lattice & 4 & Kotay et al., 1998 [6] \\
\hline Polybot & Chain & 2 & Castano et al., 2002 [25] \\
\hline Metamorphic & Chain & 1 & Golovinsky et al., 2004 \\
\hline Telecube & Lattice & 6 & Suh et al., 2002 \\
\hline I-Cube & Lattice & 3 & Ünsal et al., 2001 [22] \\
\hline Pneumatic & Lattice & 2 & Inou et al., 2002 \\
\hline Uni Rover & Mobile & 2 & Damoto et al., 2001 \\
\hline M-TRAN & Hybrid & 2 & Murata et al., 2002 [28] \\
\hline Atron & Lattice & 1 & Brandt et al., 2007 [20] \\
\hline Swarm-bot & Mobile & 3 & Groß et al. [11] \\
\hline Superbot & Hybrid & 3 & Rubenstein et al. [27] \\
\hline Molecube & Chain & 1 & Studer and Lipson, 2006 \\
\hline Miche & Lattice & 0 & Gilpin et al., 2008 \\
\hline $\mathrm{ACM}$ & Chain & Various & Hirose and Mori, 2004 \\
\hline Miniturized & Hybrid & 0 & Tomita et al. [14] \\
\hline Fractum & Lattice & 2 & Tomita et al. [14] \\
\hline M-TRAN II & Hybrid & 2 & Murata $[21,28]$ \\
\hline
\end{tabular}

a dsPIC 30F6014A microcontroller with $144 \mathrm{~KB}$ of program memory and $8 \mathrm{~KB}$ of RAM. E-Puck has eight IR sensors for measuring proximity to objects and for measuring ambient light. It has a speaker for audible feedback, three-directional microphones that can be used for sound localization, and a 3-axis accelerometer. The robot has a color camera, a number of LEDs to signal/show its state, and Bluetooth for its main wireless communication channel. The robots can be programmed via the Bluetooth communication channel.

Table 1 lists a number of self-reconfigurable robots, their classification, and source of relevant reference information.

\section{Hardware Architecture}

The hardware of robot swarms consists of a broad range of components, including a wide variety of sensors, actuators, controllers, and cameras. It is common practice to use hardware customized for specific applications, resulting in an increased degree of heterogeneity which in turn results in increased complexity for software developers. The nature of the tasks and the field of application influence the hardware architecture of a swarm robot, which must have the ability to navigate in dynamically changing environments without being third-party interaction, human, or otherwise. The choice of appropriate sensors in robot swarms helps the individual robots to perceive the various physical properties of their surroundings. Based on measured data, the swarm robots may conclude that one or more particular actions are necessary based on their current state. They will then activate and control actuator devices to interact with and influence their environment. In this section, we review a variety of hardware architectures for swarm robots based sensory platform, actuation, locomotion, controller, and power supply.

3.1. Sensors Platform Review. Sensors are used to provide information about the surrounding environment to the controller-a process known as mapping. In swarm robotics, sensors are used to detect obstacles, to find targets, to find paths, and for communication. There are many different types of sensors used for swarm robots, but the IR Proximity Sensor $[4,7-13,16,17,27,34]$ is most commonly used, because it is a chip, small in size, easy to mount, and able to detect objects at a distance of $5 \mathrm{~cm}$ to $15 \mathrm{~cm}$ depending on the color of object. Such an IR sensor is shown in Figure 4. An IR proximity sensor works by applying voltage to a pair of IR light emitting diodes, in response to which they emit infrared light which propagates through the air. Once the emitted light hits or is blocked by an object, it reflects back to the sensor. The closer the object, the stronger the intensity of reflected light will be. Lee and Chong [40] addressed practical design and hardware implementation of DRIr (Dual Rotating Infrared Sensor) proximity sensors for mobile robot swarms. These sensors are characterized by low cost, high reliability, and easy integratability into commercial mobile robots. The DRIr also provides robots with full 360 degree azimuth scanning and controllable range-tracking capabilities. Another type of sensor used in swarm robots is the Laser Range Finder (LRF) sensor, which has higher speed, accuracy, and resolution than LED-based IR sensors. LRF sensors have been used in various applications of mobile robots, but such applications are limited due to the high expense of LRF compared to other proximity sensing techniques [41]. Another type of proximity sensor is the Sonar or Ultrasonic sensor [42, 43], 


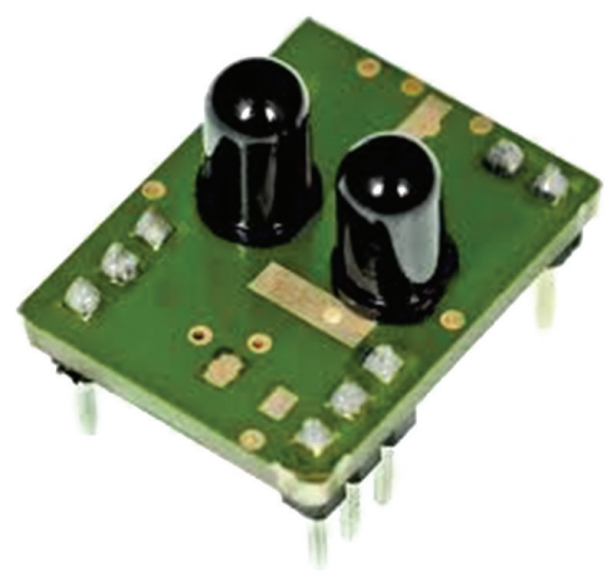

FIGURE 4: IR proximity sensor module.

providing a mobile Ultrasonic Relative Positioning System (URPS) that can be used by robots to detect the distances and angles of surrounding robots in relation to each other. Sonar time-of-flight distance sensor measurements work over a longer range than infrared sensors but can be easily affected by the hardness of objects, which can result in undesired measurement variation due to differences in how sonar waves are reflected and refracted according to varying surface properties.

Some swarm robots use a vision system such as a camera to determine the position of other swarm robots as well as for path finding and localization $[33,34]$. The S-Bot (Swarm Bot) uses a VGA-resolution, omnidirectional camera for visual communication with other robot units and to determine the position of a target for long and short distance sensing. LEDs of different colors are used for visual signaling with other robots. In some of the swarm robot modules [44], omnidirectional microphones, humidity sensors, temperature sensors, axis accelerometers, incremental encoders, and torque sensors are used. Sometimes odometry sensors are also used to aid in exploring all of the positions of swarm modules in a working environment.

3.2. Actuation and Locomotion Platform Review. The goal of a fully autonomous swarm robot team is to self-navigate, grasp objects, and physically interconnect with each other to accomplish self-reconfiguration, self-reassembly, and selfreplication by means of a gripper or manipulator. Another goal is the transport of a heavy object from one location to another location in any type of terrain with the help of locomotion units such as wheels, tracks, treels (track/wheel combinations), or legs (quadrupedal, hexapedal, etc.). Sensors and actuators must be selected and designed while considering constraints such as power consumption, voltage, driving signals (ideally pure digital), size, and cost.

An artificial localization of swarm robots is mainly classified into two categories: absolute positioning and relative positioning [22]. In some swarm robots, GPS (Global Positioning System) is used to navigate in an unexplored environment. GPS consists of a number of satellites (originally 24 , currently 32 ) in earth's orbit, each transmitting time and position information that can be used by any receiver on or near the earth with an unobstructed view of at least four satellites to determine its position and altitude. The robot swarm can use the trilateration method to calculate an absolute location with a predetermined accuracy error. The accuracy error, the group has deemed, is not critically important since the robots can communicate with each other, permitting them to determine a relative location with respect to one another. When they localize with each other, the searching algorithm allows each robot to cover more area with much more efficiency. However, even though using GPS for determining absolute position is relatively expensive, another simple localization technique known as odometry is commonly used. This technique is accurate in the short term and inexpensive, using wheel revolution data to determine linear displacement relative to the floor. The drawback to this technique is that it is highly sensitive to error. That is, if there is a slight error in calculation, then the entire set of location calculations is skewed. Servo motors are used for locomotion in the swarm robots in addition to an incremental encoder or odometry unit. The actuation modules are of the following types.

3.2.1. Wheeled Swarm Robot. This swarm robot module might have two wheels for locomotion driven by servo motors. Most mobile robots only provide simple motion control by switching the DC servo motors on and off. E puck [39], Alice [38], and Sumobot use a two-wheel robot module, while SamBot [33] maneuvers by means of a multicrawler robot created by self-assembly. The three-wheel [31] and Boe bot platforms are also used in swarm robots, with gear assembly attached with a DC motor. The shape of the platform might be triangular or circular. Between 1995 and 1997, Takeshi Aoki, Yuki Murayama, and Shigeo Hirose [45] built an omnidirectional, three-wheel planetary exploration robot, the Tri-Star. The chassis is deployed at the exit of the container and the wheels are expandable.

Some swarms use four wheels for movement and locomotion. The omnidirectional mobile robot described in [46] is equipped with four independent driving wheels equally spaced at 90 degrees from one another. The drawback of having a wheeled robot is that if any obstacle comes in the way of the robot, the robot may not be able to run over that obstacle. Also, the speed of a wheeled robot changes with changes in surface roughness and inclination. However, wheeled robots require little power and are energy efficient.

3.2.2. Tracked Swarm Robot. Tracked robots use crawl units or tracks similar to those used for terrestrial mobile applications like military tanks and automobiles. These tracks are especially suited for motion on difficult terrain. The robot Aurora Automatika in Pennsylvania, built by Hagen Schempf in 1999, consists of a single and directional track. Researchers at the University of Wuerzburg built a two-tracked Nanokhod robot, with an articulated pendulum used as weight cons and itself made of a caterpillar. It can move horizontally on 


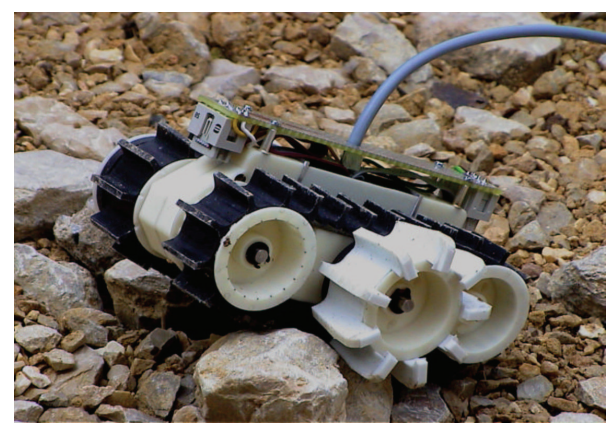

FIGURE 5: S-Bot Tracks [44].

slopes. The Nanokhod [47] is a miniaturized track-enabled robot that was developed based on Russian technology. The tracker consists of two "caterpillar" track units, a tether unit, and a payload cabin. The caterpillar tracks are driven by four internal drive units, each consisting of a stepper motor attached to a $64: 1$ planetary gear in front of a crown and pinion stage. The output stage is a miniaturized harmonic drive whose input is coupled directly to the crown gear. The omnidirectional mobile robot is equipped with four independent driving wheels equally spaced at 90 degrees from one another. The tracked robot has better traction capability on loose soil and can handle large hinder and small holes, but it is inefficient due to the friction of tracks that "scrub" along surfaces while turning.

3.2.3. Leg-Based Robot. Some swarm robots use legs for locomotion, but they are very complex to build and controlling the legs is also complicated. They tend to be very slow and create an impact with each step.

3.2.4. Hybrid Robot. A main premise behind hybrid robot architecture is that the combination of any two mechanisms is better than a single one, as it benefits from the advantages of the two. This concept is highly used in recent prototypes such as the Swarm Bot [34] and S-Bot [44]. The S-Bot is based on track and wheel combination platforms called Treels, as shown in Figures 5 and 6. Each treel is controlled by an independent motor so that the S-Bot can freely move in any environment and can easily rotate on a spot. This mechanism allows each S-Bot to move over moderately rough terrain with complex obstacles. AutoBot [32] uses a differential drive with reliable motion control configured with caster wheels and a pulse width modulation technique that is employed for DC motor control.

Piezoelectric actuators are commonly used for locomotion and actuation of mobile microrobots of a size between $1 \mathrm{dm}^{3}$ and $1 \mathrm{~cm}^{3}$.

Figure 7 shows the Pioneer II P2AT-8 robots and custombuilt track robot at ACE Lab, UTSA. In this module, a sonar based sensor platform is used with both four-wheeled robots and tracked robots.

Manipulation of objects by swarm robots is accomplished by grasping, pushing, and caging [48]. Grasping action includes form closure and force closure techniques. By way

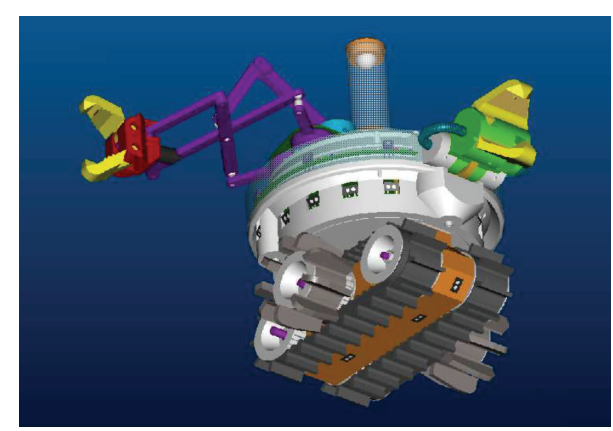

FIGURE 6: Bottom view of the S-Bot robot showing its independently controlled treels [44] (copyright @ 2002 Mondada et al.).

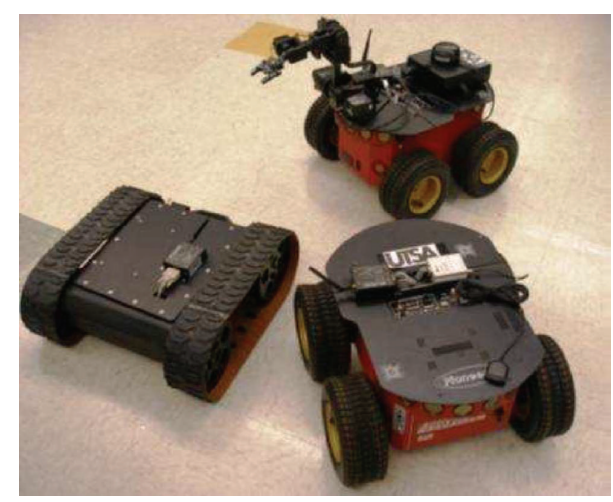

FIgURE 7: Pioneer II P2AT-8 (copyright @ 2007 Pioneer Inc., et al.).

of example, grippers $[33,44,47]$ are used as manipulators in almost all swarm robots, both for interconnection with other swarm robots and for grabbing (grasping) objects. Such grippers are usually operated by a DC motor. Opening and closing of the grippers are typically measured by means of an optical sensor. In Voyles' TerminatorBot [49], the robot consists of a cylindrical body with dual 3-degree-of-freedom (DOF) arms controlled by two gear motors that can be fully stowed inside the body of the robot. Some robots use a mechanical hook controlled by a spring return actuator to connect with other robots. Minghui et al. [50] describe a wheel-manipulator robot consisting of a triangle wheel and a 5-DOF arm with an end effector. Other connection techniques include point-to-point and surface-to-surface attachment mechanisms. M-TRAN, CORNO, and I-CUBE [19] use a surface-to-surface connection in which an active attachment-making connector extends three hooks from its mating surface to grab onto features of a passive mating connector. The passive connectors are built from two bars of stainless steel rigidly integrated in the hemisphere. These three hooks are driven by a DC motor via a worm gear. MiLyBot [51] uses a Solarbotics motor, as shown in Figure 8. These motors exhibit low power consumption and excellent torque.

3.3. Controller and Communication Module Platform Review. In robot swarms, communication can work using any of several different techniques, depending upon factors like 


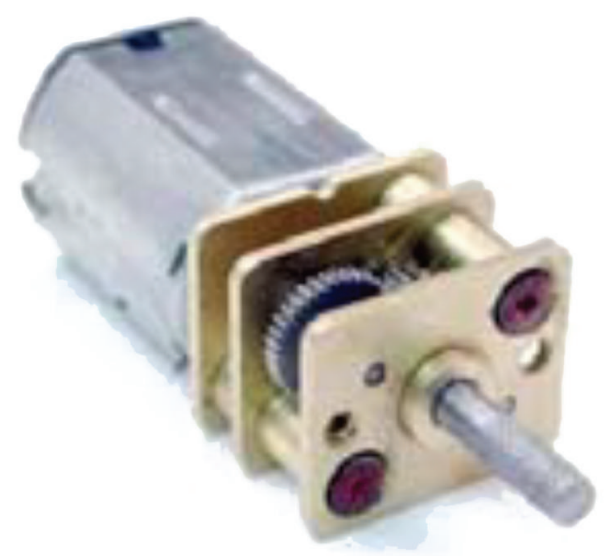

Figure 8: Solarbotics motor and gearbox assembly used in MiLyBot [51] (copyright @ 2008 Luis Vegas et al.).

robot size, robot cost, budget, the environment in which the robots will work, and other application-specific limitations. Generally speaking, swarm robots are controlled by one of two broad approaches: a centralized approach where a single supervisory robot plans for the group, or a distributed approach where each robot is responsible for its own planning [52]. In recent years, robots have become more mobile, requiring wireless communication techniques like Bluetooth, wireless LAN, stigmergy, or visual signaling using IR LEDs. "Stigmergy" was introduced by Pierre-Paul Grasse in the late 1950 s to describe the type of indirect communication employed by insect life such as ants and termites, using pheromones to mark the shortest path back to the nest, to mark the location of food sources, or to identify danger. However, communication can also be established by sending messages to other robots using Bluetooth, wireless LAN, or infrared LEDs. Infrared LEDs are used in SWARM BOT; S-BOT achieves visual communication with different color LEDs and a camera mounted on the top of robot to receive signals from other robots. This technique is very economical and easy to install on minirobots or swarm robots, but sunlight or other light sources can interfere with this type of communication. Wireless LANs may be used on midsized robots to send high-volume message traffic to the robot team, but this type of network can be disturbed by other RF-radiating devices. One of the best and most costeffective techniques for communicating with team swarms is Bluetooth communication, which requires a unique ID for each swarm robot. There are numerous Bluetooth devices or cards available on the market, [31] using both WaveLAN and Bluetooth wireless communication systems with wireless antennae.

$\mathrm{Li}$ et al. [53] explored the use of wireless mesh networks (WMNs) and mobile ad hoc networks (MANETs) for robot communication with the help of mesh routers, PDAs, wireless adapters, and GPS on each robot. In CORNO [7], each module communicates with other modules by means of an IR transmitter and receiver to form a local communication network. CORNO is controlled by a BASIC STAMP 2 processor card. M-TRAIN [28] also uses the BASIC
STAMP 2 microcontroller for controlling the modules and communicating through the Relay PIC by serial communication. AUTOBOT [32] uses $2.4 \mathrm{GHz} 1 \mathrm{Mbps}$ GFSK radiobased local communication by means of a Cypress $\mathrm{CyFi}$ CYRF7936 radio integrated circuit and an integrated PCB Trace antenna. In a swarm robotics project by Nataraj et al. [54] at Villanova University, Pennsylvania employed LEGO NXT mobile robots with Bluetooth communication via NXT bricks, with each containing an Atmel 32-bit ARM7 processor running at $48 \mathrm{MHz}$ with $64 \mathrm{~KB}$ of RAM and $256 \mathrm{~KB}$ bytes flash memory. Communication between the NXT robots and a PC laptop host was implemented using a D-Link DBT-120 wireless Bluetooth 2.0 USB Adapter.

The Autonomous Miniature mobile Robot (AMiR) [55] uses an AVR microcontroller series as the main processor for managing all AMiR's modules. The micro-controller ATMEGA168, clocked at $8.0 \mathrm{MHz}$ using its internal RC oscillator and IR based local communication for each module. At Cleveland State University [4] in the summer of 2007, square robot swarms were designed with communication between these robots and the base station accomplished using a MaxStream 9Xtend RF transmitter/receiver and the PIC18F4520 as microcontroller with C language complier. KOBOT [56] was designed as a self-organized flocking robot using an IEEE 802.15.4/ZigBee compliant XBee wireless module with a range of approximately $20 \mathrm{~m}$ for communication between robots, a PIC 18F4620A microcontroller, and a PC (supervisor). There are also other commercially available lowcost microcontroller devices available such as the Arduino, which is a flexible, open source electronic platform that is easy to use and easy to program in a variety of programming languages. Wireless communication can also be established using CC250 with the Atmega16 built-in Universal Asynchronous Receiver Transmitter (UART).

3.4. Power Option. Another important consideration for swarm robots is the power supply, since each swarm robot is very small and highly mobile in nature, suggesting that the power supply should be small and light enough to be mounted on the robot. Most swarm robots work on 5 to $25 \mathrm{~V}$ DC power supplied by rechargeable lithium batteries. Lithium-Polymer batteries (Li-Po) [57] have several advantages in such applications, including high energy density, thin size, and operational safety when compared to other rechargeable batteries. The ATRON swarm robot module, $11 \mathrm{~cm}$ in diameter, equips each module with two $3.6 \mathrm{~V}$ $980 \mathrm{mAh}$ ion-lithium-polymer cells. This provides 7.2 volts at an ampacity of $980 \mathrm{mAh}$ for each module. S-Bot is equipped with two Lithium-ION batteries placed between the tracks. The power storage capacity of these batteries is $10 \mathrm{Wh}$. Preliminary measurements show a power consumption for one S-Bot, between 3 and $5 \mathrm{~W}$, which ensures continuous operation for at least two hours.

AutoBot is powered by an $11.1 \mathrm{v}$ Li-Po battery with $500 \mathrm{mAh}$ ampacity. CYBOTS are smaller in size, around $25 \mathrm{~cm}$ in length, and use a pair of Li-Po rechargeable battery as power source. 
TABLE 2: Advantages and disadvantages of various swarm robot platforms.

\begin{tabular}{|c|c|c|c|}
\hline Sr. no. & System & References & Advantages and Disadvantages \\
\hline 1 & PolyBot & $\begin{array}{c}\text { Yim et al. } \\
{[3,3,12,13,23,24]}\end{array}$ & $\begin{array}{l}\text { Advantages: 1st system to demonstrate the ability of self-reconfiguration with most active } \\
\text { modules in a connected system. Each module fits within the } 5 \mathrm{~cm} \text { cube. They are versatile in } \\
\text { nature. Each module contains a Motorola PowerPC } 555 \text { processor with } 1 \text { MByte of external } \\
\text { RAM and DC brushless motor with built in hall effect sensors. } \\
\text { Limitation: insufficient sensory unit for mapping of environment. PolyBot cannot work in } \\
\text { unknown environment with rough surface or when obstacle avoidance is not possible. }\end{array}$ \\
\hline 2 & M-TRAN & Tomita et al. [14] & $\begin{array}{l}\text { Advantages: very small actuated modules, highly robust, miniature, and reliable. Quick } \\
\text { self-reconfiguration and versatile robotic motion. } \\
\text { Limitations: connection mechanism works on an internally balanced magnetic field that is } \\
\text { not strong enough to hold the other modules. Single M-TRAN module does not have } \\
\text { enough DOFs for switching from one posture to another form. Lack of sensors leads to } \\
\text { mapping and control problems. Power consumption is more as it uses servo motor and } \\
\text { electromechanical force for connectivity. }\end{array}$ \\
\hline 3 & ATRON & Støy et al. $[7,15]$ & $\begin{array}{l}\text { Advantages: each module is equipped with its own power supply, sensors, and actuators, } \\
\text { allowing each module to connect and communicate with a neighbor module. Each module } \\
\text { is able to sense the state of its connectivity and relative motion. } \\
\text { Limitation: since each module includes two-axis accelerometers only, a module cannot tell if } \\
\text { it is turned upside down or not. When two modules are connected, it is very difficult for } \\
\text { them to move themselves, which requires cooperation from its neighbor. They are not } \\
\text { mechanically stable and due to this mechanical instability, their electronic performance is } \\
\text { poor. }\end{array}$ \\
\hline 4 & SamBot & Wei et al. [33] & $\begin{array}{l}\text { Advantages: SamBot is a combination of mobile and chain-based modules capable of } \\
\text { self-assembly and self-reconfiguration. SamBot uses } 4 \text { docking mechanisms for connecting } \\
\text { with other SamBots. Detects other SamBots using infrared sensors. } \\
\text { Limitation: infrared sensors limit the search range and require line of sight between } \\
\text { SamBots. SamBot architecture lacks extra actuators, grippers, and sensors for gathering } \\
\text { information about the working environment. }\end{array}$ \\
\hline 5 & $\begin{array}{l}\text { Swarm Bot } \\
\text { (S-Bot) }\end{array}$ & $\begin{array}{c}\text { Mondada et al. } \\
{[35,44,57]}\end{array}$ & $\begin{array}{l}\text { Advantages: robot swarms consisting of } 2 \text { to } 40 \text { S-bots have been successfully demonstrated. } \\
\text { S-Bots are fully autonomous mobile robots capable of self-navigation, perception of the } \\
\text { environment and object. Capable of communicating other S-Bots and transporting of heavy } \\
\text { objects over very rough terrain. } \\
\text { Limitations: initial cost is high. Images and sound are the only way of communicating with } \\
\text { other S-Bots. The large number of sensors and actuators consumes power, reducing } \\
\text { functionality and operating time. }\end{array}$ \\
\hline 6 & CONRO & Støy et al. $[7,15]$ & $\begin{array}{l}\text { Advantages: small, rectangular, self-reconfigurable swarm robot with a low price. Versatile. } \\
\text { limitation: uses onboard low-capacity batteries that limit the usefulness of modules. Limited } \\
\text { sensors limit ability to sense surroundings. Only two controllable degrees of freedom. }\end{array}$ \\
\hline 7 & MiLyBots & Vega et al. [51] & $\begin{array}{l}\text { Advantages: low cost, reliable, robust, reusable, movable, size-efficient, power sparing, } \\
\text { wireless, dynamically programmable swarm robots. } \\
\text { Limitation: MiLyBots are not self-reconfigurable, self-assembled swarm robots. Lack } \\
\text { actuators and connection mechanisms for physically attaching to other modules. }\end{array}$ \\
\hline 8 & I-Cube & Ünsal et al. [22] & $\begin{array}{l}\text { Advantages: I-Cubes are low cost, small lattice based swarm robot with } 3 \text { DOF. } \\
\text { Limitation: unable to provide heavy object transport. Limited sensors. Lacks actuator } \\
\text { mechanism. }\end{array}$ \\
\hline
\end{tabular}

\section{Hardware Design Challenges}

The performance of any machine or interoperable group of machines is highly dependent on hardware architecture, that is, on the overall mechanical and electronic control design and structure. Over the past two decades, numerous hardware architectures have been designed and developed for self-reconfigurable, self-replicating, and self-reassembling swarm robots. Each structure has focused on a different set of factors such as flexibility, degrees of freedom, torque to weight ratio, power consumption, cost, size, and control mechanism.
However, there are some fundamental inherent limitations imposed by various architectures that can have a profound effect on how control and manipulation of autonomous mobile swarms are accomplished. These architectural limitations can affect the precision of robot movement, robot strength, and the ruggedness of docking interfaces between modules. Motor power, power management, and the speed with which individual modules can move are also limiting factors on the performance of swarm reconfigurable robots.

Table 2 lists a number of swarm robot systems, along with their advantages, disadvantages, and limiting factors. 
In Table 2, all of the swarm robot systems are homogeneous except S-Bot, in which the swarm robots are heterogeneous.

\section{Conclusion}

In this survey, we have outlined a number of aspects of swarm robot hardware architecture, focusing on the types of interactions that can occur in such systems, including self-reconfigurability, self-assembly, and self-replication. To explore the challenges related to swarm robot systems, we have outlined a general classification framework for considering the various design and protocol aspects that can be used to develop specific applications of swarm robotics. One of the challenges faced by swarm system designers is the selection of an appropriate swarm architecture to best address the specific constraints in real-world applications. Cost and miniaturization/size are always extremely important factors for swarm robots.

In our future work, we will design and build a mediumpowered hybrid mobile swarm robot system that is cheap enough to mass produce. Numerous researchers have faced and described significant challenges, and while some progress has been made to overcome these problems, there is still considerable work to be done.

\section{Conflict of interests}

The authors declare no conflicts of interest and have no direct financial relation with the commercial identity mentioned in the paper.

\section{References}

[1] A. J. C. Sharkey, "Robots, insects and swarm intelligence," Artificial Intelligence Review, vol. 26, no. 4, pp. 255-268, 2006.

[2] M. G. Hinchey, R. Sterritt, and C. Rouff, "Swarms and swarm intelligence," IEEE Computer, vol. 40, no. 4, pp. 111-113, 2007.

[3] M. Yim, W. M. Shen, B. Salemi et al., "Modular selfreconfigurable robot systems [grand challenges of robotics]," IEEE Robotics and Automation Magazine, vol. 14, no. 1, pp. 4352, 2007.

[4] G. Bekey and J. Yuh, "Reviewing the issues of robotic self-X," IEEE Robotics and Automation Magazine, vol. 14, no. 4, pp. 6-7, 2007.

[5] H. Bojinov, A. Casal, and T. Hogg, "Emergent structures in modular self-reconfigurable robots," in Proceedings of the IEEE International Conference on Robotics and Automation (ICRA '00), pp. 1734-1741, San Francisco, Calif, USA, April 2000.

[6] K. Kotay, D. Rus, M. Vona, and C. McGray, "Self-reconfiguring robotic molecule," in Proceedings of the IEEE International Conference on Robotics and Automation, pp. 424-431, Leuven, Belgium, May 1998.

[7] K. Støy, W. M. Shen, and P. Will, "How to make a selfreconfigurable robot run," in Proceedings of the 1st International Joint Conference on Autonomous Agents and Multiagent Systems (AAMAS '02), pp. 813-820, ACM, New York, NY, USA, 2002.

[8] J. von Neumann and A. W. Burks U, Theory of Self-Reproducing Automata, University of Illinois Press, Urbana, Ill, USA, 1962.
[9] J. Suthakorn, "Toward self-replicating robots: an overview on self-replication machine," Journal of Thai Robotics Society, vol. 3, no. 1, pp. 66-80.

[10] K. Lee and G. S. Chirikjian, "Robotic self-replication," IEEE Robotics and Automation Magazine, vol. 14, no. 4, pp. 34-43, 2007.

[11] R. Groß, E. Tuci, M. Dorigo, M. Bonani, and F. Mondada, "Object transport by modular robots that self-assemble," in Proceedings of the IEEE International Conference on Robotics and Automation (ICRA '06), pp. 2558-2564, Orlando, Fla, USA, May 2006.

[12] M. Yim, Y. Zhang, and D. Duff, "Modular robots," IEEE Spectrum, vol. 39, no. 2, pp. 30-34, 2002.

[13] M. Yim, K. Roufas, D. Duff, Y. Zhang, C. Eldershaw, and S. B. Homans, "Modular reconfigurable robots in space applications," in Proceedings of the International IEEE Conference on Robotics and Automation, pp. 225-237, 2003.

[14] K. Tomita, S. Murata, H. Kurokawa, E. Yoshida, and S. Kokaji, "Self-assembly and self-repair method for a distributed mechanical system," IEEE Transactions on Robotics and Automation, vol. 15, no. 6, pp. 1035-1045, 1999.

[15] K. Støy, W. M. Shen, and P. Will, "On the use of sensors in selfreconfigurable robots," in Proceedings of the 7th International Conference on Simulation of Adaptive Behavior on from Animals to Animats (ICSAB '02), pp. 48-57, Cambridge, Mass, USA, 2002.

[16] F. Ducatelle, G. A. Di Caro, and L. M. Gambardella, "Cooperative self-organization in a heterogeneous swarm robotic system," Dalle Molle Institute for Articial Intelligence Galleria 2, Manno, Switzerland.

[17] E. Mytilinaios, M. Desnoyer, D. Marcus, and H. Lipson, "Designed and evolved blueprints for physical self-replicating machines," in Proceedings of the 9th International Conference on the Simulation and Synthesis of Living Systems (Artificial Life IX), pp. 15-20, Cambridge, Mass, USA, 2004.

[18] V. Zykov, E. Mytilinaios, B. Adams, and H. Lipson, "Robotics: self-reproducing machines," Nature, vol. 435, no. 7039, pp. 163$164,2005$.

[19] M. W. Jørgensen, E. H. Østergaard, and H. H. Lund, "Modular ATRON: modules for a self-reconfigurable robot," in Proceedings of the IEEE/RSJ International Conference on Intelligent Robots and Systems (IROS '04), pp. 2068-2073, Sendai, Japan, October 2004

[20] D. Brandt, D. J. Christensen, and H. H. Lund, "ATRON robots: versatility from self-reconfigurable modules," in Proceedings of the IEEE International Conference on Mechatronics and Automation (ICMA '07), pp. 26-32, Harbin, China, August 2007.

[21] S. Murata, E. Yoshida, H. Kurokawa, K. Tomita, and S. Kokaji, "Self-repairing mechanical systems," Autonomous Robots, vol. 10, no. 1, pp. 7-21, 2001.

[22] C. Ünsal, H. Kiliççöte, and P. K. Khosla, "Modular selfreconfigurable bipartite robotic system: implementation and motion planning," Autonomous Robots, vol. 10, no. 1, pp. 23-40, 2001.

[23] M. Yim, Y. Zhang, J. Lamping, and E. Mao, "Distributed control for 3D metamorphosis," Autonomous Robots, vol. 10, no. 1, pp. 41-56, 2001.

[24] M. Yim, D. G. Duff, and K. D. Roufas, "PolyBot: a modular reconfigurable robot," in Proceedings of the IEEE International Conference on Robotics and Automation, pp. 514-520, IEEE Computer Society Press, San Francisco, Calif, USA, 2000. 
[25] A. Castano, W. M. Shen, and P. Will, "CONRO: towards deployable robots with inter-robot metamorphic capabilities," Autonomous Robots, vol. 8, no. 3, pp. 309-324, 2000.

[26] A. Castano, A. Behar, and P. M. Will, "The Conro modules for reconfigurable robots," IEEE/ASME Transactions on Mechatronics, vol. 7, no. 4, pp. 403-409, 2002.

[27] M. Rubenstein, K. Payne, P. Will, and W. M. Shen, "Docking among independent and autonomous CONRO selfreconfigurable robots," in Proceedings of the IEEE International Conference on Robotics and Automation, pp. 2877-2882, Los Alamitos, Calif, USA, May 2004.

[28] S. Murata, E. Yoshida, A. Kamimura, H. Kurokawa, K. Tomita, and S. Kokaji, "M-TRAN: self-reconfigurable modular robotic system," IEEE/ASME Transactions on Mechatronics, vol. 7, no. 4, pp. 431-441, 2002.

[29] T. Fukuda and S. Nakagawa, "A dynamically reconfigurable robotic system (concept of a system and optimal configurations)," in Industrial Applications of Robotics and Machine Vision (IECON '87), vol. 0856 of Proceedings of SPIE, Cambridge, Calif, USA, 1987.

[30] T. Fukuda, S. Nakagawa, Y. Kawauchi, and M. Buss, "Structure decision method for self organising robots based on cell structures-CEBOT," in Proceedings of the IEEE International Workshop on Intelligent Robots, Los Alamitos, Calif, USA, 1988.

[31] K. A. Hawick, H. A. James, J. E. Story, and R. G. Shepherd, An Architecture for Swarm Robots, Computer Science Division, School of Informatics University of Wales, North Wales, UK.

[32] M. Gupta and K. Singh, "AutoBot: a low cost platform for swarm research applications," in Proceedings of the 3rd International Conference on Emerging Trends in Engineering and Technology (ICETET '10), pp. 33-36, Goa, India, November 2010.

[33] H. Wie, Y. Cai, H. Li, D. Li, and T. Wang, "Sam-bot: a selfassembly modular robot for swarm robot," in Proceedings of the IEEE International Conference on Robotics and Automation (ICRA '10), pp. 66-71, Anchorage, Alaska, USA, May 2010.

[34] M. Dorigo, "SWARM-BOT: an experiment in swarm robotics," in Proceedings of the IEEE Swarm Intelligence Symposium (SIS '05), pp. 192-200, June 2005.

[35] F. Mondada, A. Guignard, M. Bonani, D. Bär, M. Lauria, and D. Floreano, "SWARM-BOT: from concept to implementation," in Proceedings of the IEEE/RSJ International Conference on Intelligent Robots and Systems, pp. 1626-1631, October 2003.

[36] D. Y. James McLurkin, "Dynamic task assignment in robot swarms," in Proceedings of the Robotics: Science and Systems, Massachusetts Institute of Technology, Computer Science and Artificial Intelligence Lab, Cambridge, Mass, USA, 2005.

[37] G. Caprari and R. Siegwart, "Mobile micro-robots ready to use: alice," in Proceedings of the IEEE IRS/RSJ International Conference on Intelligent Robots and Systems (IROS '05), pp. 3845-3850, Edmonton, Canada, August 2005.

[38] W. C. Evans, G. Mermoud, and A. Martinoli, "Comparing and modeling distributed control strategies for miniature selfassembling robots," in Proceedings of the IEEE International Conference on Robotics and Automation (ICRA '10), pp. 14381445, Anchorage, Alaska, USA, May 2010.

[39] C. M. Cianci, X. Raemy, J. Pugh, and A. Martinoli, "Communication in a swarm of miniature robots: the e-puck as an educational tool for swarm robotics," in Simulation of Adaptive Behavior (SAB-2006), Swarm Robotics Workshop, vol. 4433 of Lecture Notes in Computer Science, pp. 103-115, Rome, Italy, October 2006.
[40] G. Lee and N. Y. Chong, "Low-cost dual rotating infrared sensor for mobile robot swarm applications," IEEE Transactions on Industrial Informatics, vol. 7, no. 2, pp. 277-286, 2011.

[41] H. Surmann, A. Nüchter, and J. Hertzberg, "An autonomous mobile robot with a 3D laser range finder for 3D exploration and digitalization of indoor environments," Robotics and Autonomous Systems, vol. 45, no. 3-4, pp. 181-198, 2003.

[42] K. Lee and W. K. Chung, "Effective maximum likehood grid map with conflict evaluation filter using sonar sensors," IEEE Transactions on Robotics, vol. 25, no. 4, pp. 887-901, 2009.

[43] F. Rivard, J. Bisson, F. Michaud, and D. Létourneau, "Ultrasonic relative positioning for multi-robot systems," in Proceedings of the IEEE International Conference on Robotics and Automation (ICRA '08), pp. 323-328, Pasadena, Calif, USA, May 2008.

[44] F. Mondada, A. Guignard, M. Bonani, D. Bär, M. Lauria, and D. Floreano, "SWARM-BOT: from concept to implementation," in Proceedings of the IEEE/RSJ International Conference on Intelligent Robots and Systems, pp. 1626-1631, Las Vegas, Nev, USA, October 2003.

[45] T. Aoki, Y. Murayama, and S. Hirose, "Mechanical design of three-wheeled lunar rover, 'Tri-Star IV"' in Proceedings of the IEEE International Conference on Robotics and Automation (ICRA '11), pp. 2198-2203, Shanghai, China, May 2011.

[46] H. C. Huang, "Intelligent motion controller design for fourwheeled omnidirectional mobile robots using hybrid GA-PSO algorithm," in Proceedings of the IEEE International Conference on Systems, Man, and Cybernetics (SMC '11), pp. 2267-2272, Anchorage, Alaska, USA, October 2011.

[47] "Robot mobility systems for planetary surface explorationstate-of-the-art and future outlook: a literature survey," in Aerospace Technologies Advancements, D. T. T. Arif, Ed., 2010.

[48] Z. D. Wang and V. Kumar, "Object closure and manipulation by multiple cooperating mobile robots," in Proceedings of the IEEE International Conference on Robotics and Automation (ICRA '02), pp. 394-399, May 2002.

[49] R. M. Voyles, "TerminatorBot: a robot with dual-use arms for manipulation and locomotion," in Proceedings of the IEEE International Conference on Robotics and Automation (ICRA '00), pp. 61-66, April 2000.

[50] W. Minghui, M. Shugen, L. Bin, G. Haili, and W. Yuechao, "Reconfiguration optimization for a swarm of wheelmanipulator robots," in Proceedings of the IEEE International Conference on Mechatronics and Automation (ICMA '08), pp. 1035-1040, Takamatsu, Japan, August 2008.

[51] L. Vega, D. Hughes, C. Buscaron, E. M. Schwartz, and A. A. Arroyo, "MILyBots: design and development of swarm-robots," in Proceedings of the Florida Conference on Recent Advances in Robotics, 2008.

[52] M. B. de le Torre and A. Stentz, A Market Approach to Multirobot Coordination, Robotics Institute, 2001.

[53] M. Li, M. Chen, K. Lu, S. Mao, H. Zhu, and B. Prabhakaran, "Robot swarm communication networks: architectures, protocols, and applications," in Proceedings of the 3rd International Conference on Communications and Networking in China (ChinaCom '08), pp. 162-166, Hangzhou, China, August 2008.

[54] C. Nataraj, S. Reddy, M. Woods, B. Samanta, and C. Nataraj, "Swarm robotics: a research project with high school students as active participants," in Proceedings of the ASEE Annual Conference and Exposition, June 2010.

[55] F. Arvin, K. Samsudin, and A. R. Ramli, "Development of a miniature robot for swarm robotic application," International 
Journal of Computer and Electrical Engineering, vol. 1, no. 4, pp. 436-442, 2009.

[56] A. E. Turgut, H. Çelikkanat, F. Gökçe, and E. Şahin, "Selforganized flocking in mobile robot swarms," Swarm Intelligence, vol. 2, no. 2-4, pp. 97-120, 2008.

[57] F. Mondada, M. Bonani, X. Raemy et al., "The e-puck, a robot designed for education in engineering," in Proceedings of the 9th Conference on Autonomous Robot Systems and Competitions, pp. 59-65, Castelo Branco, Portugal, May 2009. 

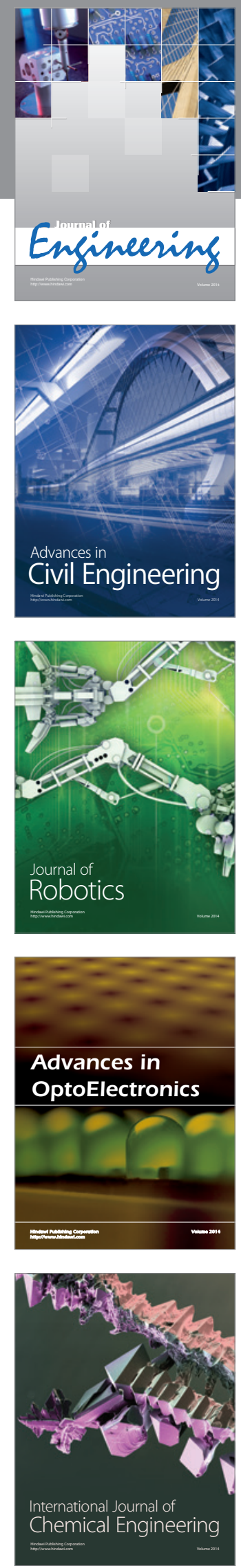

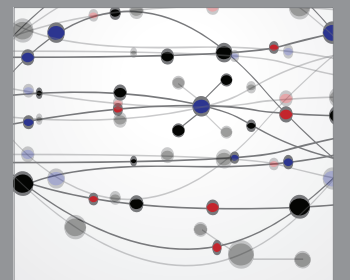

The Scientific World Journal
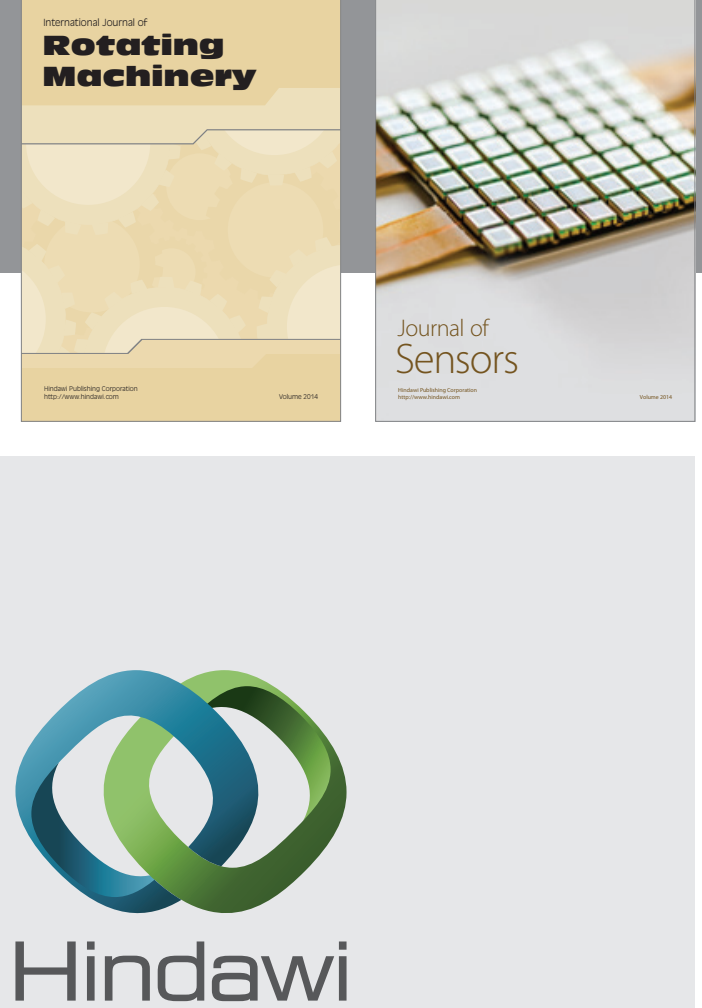

Submit your manuscripts at http://www.hindawi.com
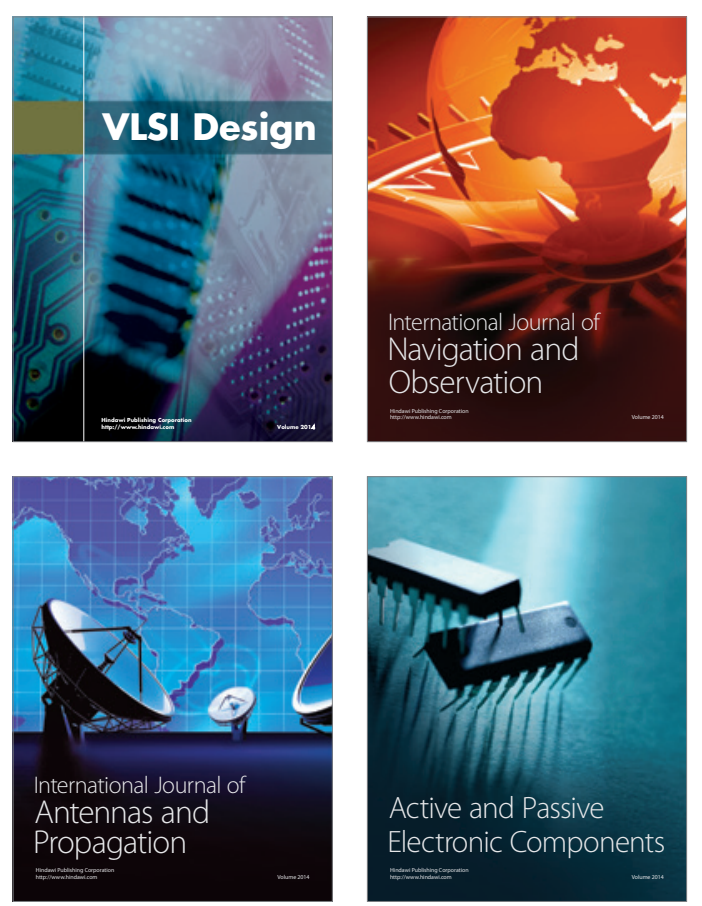
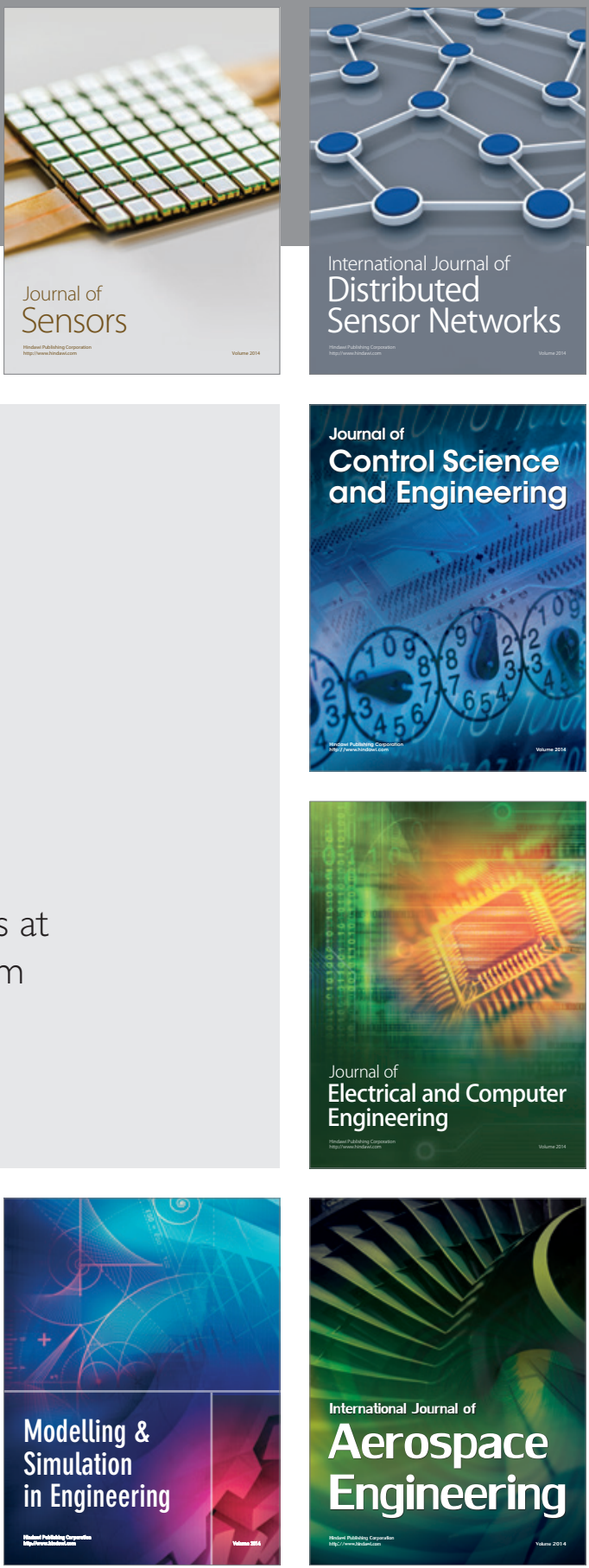

Journal of

Control Science

and Engineering
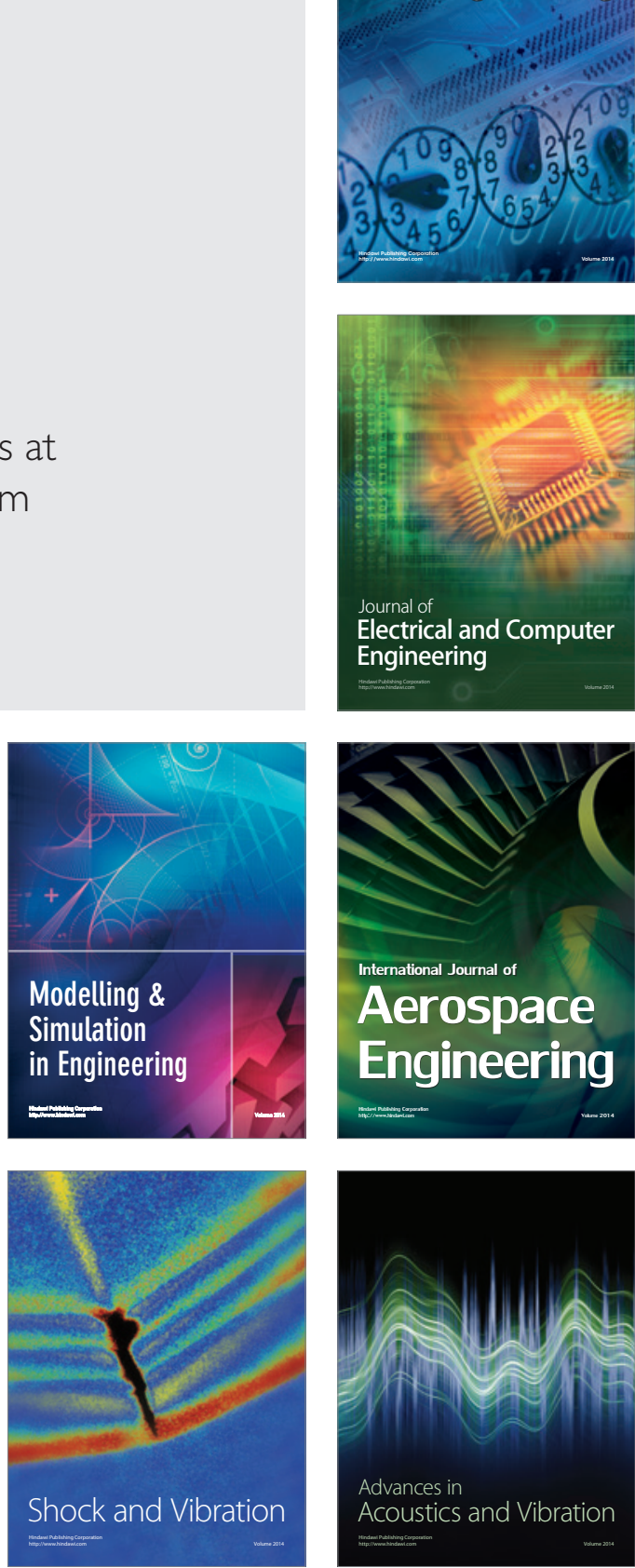